\title{
The Modelica HouseModels Library: Presentation and Evaluation of a Room Model with the ASHRAE Standard 140
}

\author{
Ana Constantin Rita Streblow Dirk Müller \\ RWTH Aachen University, Institute for Energy Efficient Buildings and Indoor Climate \\ Mathieustr. 10, 52074 Aachen
}

\begin{abstract}
As part of its contribution to IEA Annex 60, the Institute for Energy Efficient Buildings and Indoor Climate of RWTH Aachen University will make its Modelica HouseModels library available. The scope of this paper is to provide information about the library. The first part presents the library and its functionality. In the second part a room model is evaluated by using Case 600 from the test suite provided by the American Society of Heating, Refrigerating and AirConditioning Engineers (ASHRAE) in the standard 140.
\end{abstract}

Keywords: HouseModels library, IEA Annex 60, ANSI/ASHRAE Standard 140, building performance simulation

\section{Introduction}

The Institute for Energy Efficient Buildings and Indoor Climate of RWTH Aachen University aims through its research to reduce the energy consumption of buildings and increase indoor air quality, while focusing on energy generation, storage, distribution and delivery to rooms, buildings or city districts. Over the years several libraries have been developed for the dynamical simulation of building energy systems [1].

On a simulation level, modeling the thermal behavior of the construction body and the building envelope has to mediate the following trade-off: CPU(central processing unit)-time and detail. The building models serve on a basic level as an energy consumer. However through accurate modeling of heat transfer inside the building it is possible to assess the thermal comfort, while taking into account more subtle effects like the storage of energy into the thermal mass of the building. As part of its contribution to IEA Annex 60 [2] the institute will make its Modelica HouseModels library available.

The HouseModels library aims to provide standard models for one family dwellings (stand alone house), single apartments and multi-family dwellings consisting of several apartments. The particularity of this library lies in providing ready to use models for the dynamic simulation of building energy systems, while allowing for a degree of flexibility in adapting or extending these models to ones needs.

A library with models for standard houses as such does not yet exist. While at the moment the standard house models are tailor-made for the German market, it is possible to adapt them to other markets.

For the building models we used components from our Building library [1]. When setting up detailed house models, we follow an approach of building each room individually and of having each room component visible on the room model level. This approach was formed with time by dealing with project partners in our research activities and with students in our teaching activities. We analyzed how easy it is to understand the model and how quickly one can start working with it. As a consequence the graphical description of a model can quickly get cluttered, so we try to keep our detailed house models as simple as possible. An advantage of simple models with few equations is that, when set up correctly, they can lead to short CPU-times for simulations. In order to make sure that these models are correct they need to be evaluated. We choose the ANSI/ASHRAE Standard 140 [3] for evaluating or models.

Several Modelica libraries for building components already exist, some of them free [4] and [5]. While the Buildings library is already validated [6], we chose to use our own components when building the house models in order to maintain consistency between our libraries and because our models are simpler than the ones from the Buildings library. We need them as simple as possible when for example doing whole year simulations for a set up consisting of a building and its heating system, especially when the focus lies on the components in each room. For a direct comparison 
between our room model and the one from the Buildings library please refer to [4] and section 2.2 in this paper, which in order to allow for an easy comparison are similarly structured.

The first part of the paper presents the HouseModels library, while the second one presents the first steps in the ongoing work of evaluating our models with the ASHRAE Standard 140: the results for the case 600.

The library was developed using the commercial simulation environment Dymola [7].

\section{The HouseModels library}

When developing the HouseModels library we followed several goals:

- develop standard models

- model only the necessary physical processes

- build a model so that changing the parameters is easy, quick and will not lead to hidden mistakes

- have an easy to use graphical interface

- ensure a degree of flexibility for expanding or building new models

We call these house models standard for the following reasons:

- the floor layouts were made based on existing buildings, by analyzing data provided by the German Federal Statistical Office and by consulting with experts

- for modeling realistical wall structures building catalogues as well as experts were consulted

- the physical properties of the materials for the wall layers were chosen to satisfy the insulation requirements of current and past German energy saving ordinances (e.g. [8])

\subsection{Library structure}

Figure 1 presents the structure of the library. The package Rooms contains room models for one (OFD) and multi-family dwellings (MFD).

The multi-family dwelling is based on an existing building consisting of several identical apartments which is part of a larger national research project [9]. The dimensions and layout of the rooms are fixed, with

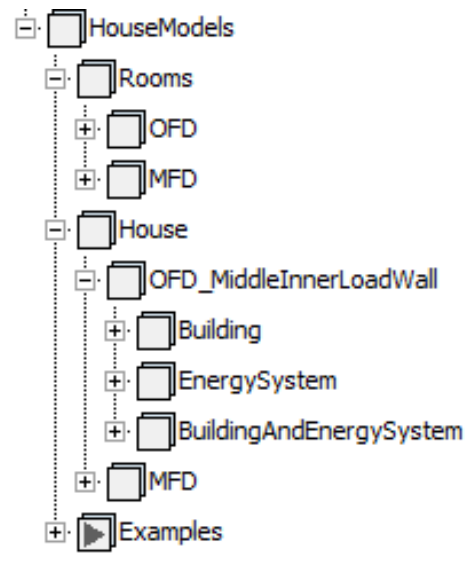

Figure 1: Structure of HouseModels library

an apartment having a living area of $70 \mathrm{~m}^{2}$ and consisting of a living room, two bedrooms, a kitchen and a bathroom.

The one family dwelling isn't based on an existing building, but on a virtual two storey building with ten rooms and a saddle roof, which is typical for German houses. The living area is $150 \mathrm{~m}^{2}$. A core of six room types was developed to model the different rooms in the house: room types with two outer walls and room types with one outer wall. Some inner walls can face just one room, while others can face two rooms. Rooms on the ground floor are connected to the ground, while rooms on the upper floor have a saddle roof. The layout of the two floors is the same. The dimensions of the room are not fixed on a room level and are set up at floor level.

The package House contains the set up house models, where the room models are connected together. The name OFD_MiddleInnerLoadWall denotes the fact that the standard house has a middle load bearing wall. Other positions of the load bearing inner wall are possible, but not included in the library. Walls are connected together and they form a room. Multiple rooms are connected together and they form a storey for the one family dwelling and an apartment for the multi-family dwelling. For the multi-family dwelling it is possible to model several storeys with apartments on top of each other, as well as several wings with apartments next to each other. Storeys are connected together to form a whole house. All connections between the connectors of the models are explicit.

For each standard house type there are packages for the building envelope (Building), for the energy system (EnergySystem) and for the building as a whole (BuildingAndEnergySystem). Currently work is be- 
ing done on the models for the energy supply systems consisting of pump, heat generator, pipes, thermostatic valves and radiators. These models are being developed starting from more detailed models to be used as a teaching tool for a course on simulation of building energy systems. They will be made available later on as part of our contribution to the IEA Annex 60.

The package Examples contains exemplary simulation setups for a room, an apartment and a one family dwelling. They can be used to learn how to set up a simulation for these models (e.g. assumption for boundary conditions for a single room) and to compare the different CPU-times for simulations using the models.

\subsection{Room model}

In a room model the following physical processes are considered:

- transient heat conduction through walls; each wall consists of several layers with different physical properties; further discretization of each layer is possible

- steady-state heat conduction through glazing systems; transmission of short wave radiation through the window depends on a constant coefficient; transmitted radiation is considered together with the radiation from room facing elements

- heat convection at outside facing surfaces either with a constant coefficient, depending on wind speed, or depending on wind speed and surface abrasiveness

- heat convection at inside facing surfaces depends on the wall orientation and the temperature difference between the room air and the wall surface

- radiation exchange between room facing elements

- temperature balance equations for the room air volume; per room only one air node is considered; humidity is not considered in the air node

The incident solar radiation on tilted surfaces is calculated using a isotropic sky model [10].

All outer walls are whole walls connected to the room air and the ambient, while inner walls are half walls, each half belonging to one of the rooms which share the wall. Airflow among rooms is not explicitly considered.

\subsection{Model parameterization}

The room model is realized by aggregating together all the components in a model, parameterizing on a room level and referencing the parameter on the component level. In this way the number of parameters is reduced, e.g. for a simple rectangular room only three parameters are needed for the dimensions of all the walls: height, length, width. On a floor level this parameterization can further be optimized, as rooms have common walls.

However not the geometrical measurements are the most problematic when parameterizing a room, but the wall types, meaning their layer structure and the physical properties of each layer. We use records for parameterizing a wall. A record contains information about the number of layers, the thickness of the layer, as well as density, thermal capacity and conductivity of each layer material together with the emissivity of the room facing layer. However setting or changing the type of each wall in each room in a house can be challenging and can lead to errors. Because we aim to build standard house models, we want to parameterize a standard house with minimal but relevant input and not have to specify each wall individually. We chose to parameterize according to the following criteria:

- thermal mass class: heavy, middle and light

- energy saving ordinance: along with the already mentioned ordinance form 2009 , older ordinances from 2002, 1995 and 1984 are considered

By specifying these two parameters, all wall, window and door types in a house are automatically set correctly.

In listing 1 an example is given of how to set up the parameter for the floor type depending on the set energy saving ordinance. As floor slabs are made of concrete for stability reasons, there is no difference between the floor types for houses with different thermal mass. As this type of coding is meant to help and not confuse a user, all these parameters are protected. The infiltration rate for a house is protected as well and depends on the energy saving ordinance. The DataBase library referenced in the listing is a library for records. The packages which are relevant for the HouseModels as well as any other relevant models, e.g. walls, windows, will also be made available.

Listing 1: Code example for setting the floor slab type (Type_FL) depending on the chosen thermal insulation 
regulation (TIR)

// Floor to ground type

parameter DataBase.Walls.GFBaseDataDefinition

Type_FL=

if $\mathrm{TIR}==1$ then

DataBase.Walls.EnEV2009.Floor.FLground_SML()

else if TIR $==2$ then

DataBase.Walls.EnEV2002.Floor.FLground_SML()

else if TIR $==3$ then

DataBase.Walls.WSchV1995.Floor.FLground_SML()

else

DataBase.Walls.WSchV1984.Floor.FLground_SML()

Furthermore for a multi-family dwelling, for each apartment, the types for floor and ceiling are automatically set if the apartment is situated on the ground, last or an arbitrary upper floor.

\subsection{Using and extending the library}

We wanted to make the library easy to use and extend by future users and developers. To this purpose we put extra effort in creating easy to understand icons and graphical interfaces for parameter input.

Figure 2 shows the needed parameters for a room type with two outer walls. The room views integrated in the parameter window help the user understand which are the width and the length of the room, where each wall type is situated and what possibilities regarding windows and doors exist for the outer walls. For each outer wall both a door and a window are possible. By checking the box for a window, element input fields for parameters are enabled. In the given example the outer wall OW1 has a window and the outer wall OW2 has a door. The already mentioned parameters for thermal mass and energy saving ordinances are visible at the top level. Other necessary parameters include the solar absorptance coefficient of the walls and the heat convection model at outside facing surfaces.

However once the parameter window is closed, the information about the geometry of the room is no longer available. Because users might want to rotate or mirror a room to build up a whole floor, we wanted to transfer the information about the position of the walls in the room, the meaning of the parameters width and length as well as the existence of windows on the icon level.

Figure 3 shows the icon for the parameterisation from figure 2. The text for the connectors was added in post-processing. However the connectors themselves have unique, easy to understand names. The information about the width and the length of the room is fixed. The thickness of the pictures for the walls indicates if

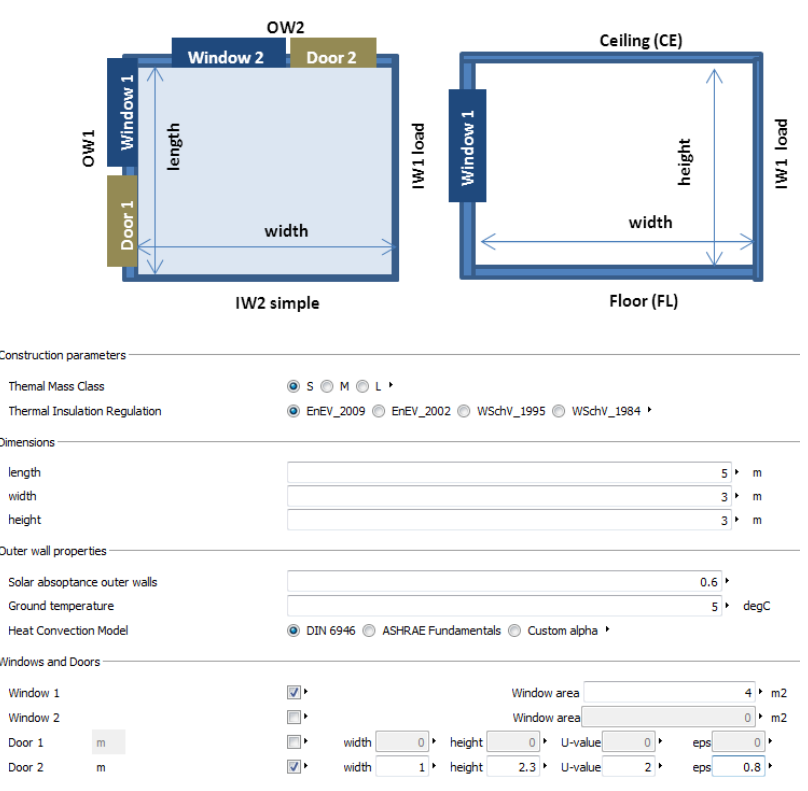

Figure 2: Parameter window for a room type

it is an inner or an outer wall. The square with the name Winl indicates the presence of a window on that wall, and the number $l$ indicates that this is wall 1 . The square is only visible if the window has been selected for the wall. In this way it is easy to combine the information given by the icon to the one which needs to be inputted in the parameter window.

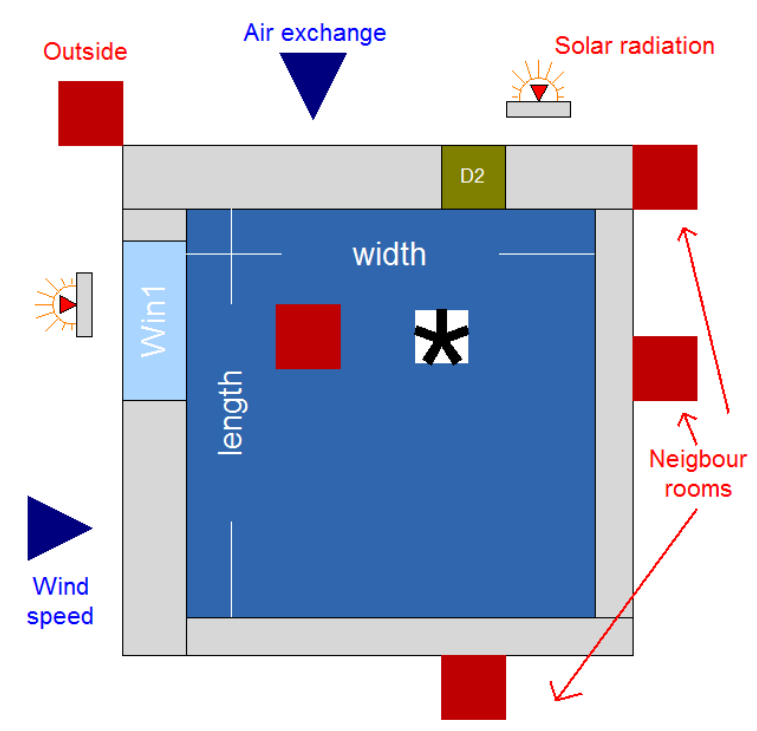

Figure 3: Icon for a room model

The set of room types developed for the one family dwelling can, if necessary, be parameterized differently than the standard model or extended in order to build up specific house models. New sets of wall, win- 
dow and door types can be developed, e.g. for older, not renovated buildings, and incorporated in the existing structure.

With the exception of the integration of images in the parameter GUI, which is done by using a Dymola specific annotation, all other annotations are independent of the used simulation environment. Also the SIunits package from the Modelica Standard Library is sufficient for using the library.

We use this library in our teaching activities as well, as it is representative of our research activities and quite complex. With the help of a custom made tutorial we help students better understand working with Modelica from a building systems performance simulation point of view. The library is planned to be made available via a dedicated website in summer 2014.

\section{Evaluation with Case 600 of ASHRAE Standard 140}

The ASHRAE Standard 140 is widely used for the evaluation of building performance simulation software especially in the English speaking community. The test suite offers a variety of tests used for validation (base cases) and for evaluation and improvement of software tools (in-depth cases). In this paper we will present the results from using a room model build with our components in the simulation setup for case 600.

Case 600 is a case which tests a low mass building without shading. The building is a rectangular room with all surfaces facing to the outside, decoupled from the ground, with a height of $2.7 \mathrm{~m}$, a length of $6 \mathrm{~m}$ and a width of $8 \mathrm{~m}$. The south facing wall has a window area of $12 \mathrm{~m}^{2}$. Detailed information about the test is provided in [3]. Once the simulation results are calculated, the way to test them is by comparison with the simulation results from other software tools, which are provided in the standard. In the following subsections the results obtained with our room model are labeled HouseModels Lib.

In this paper we will presents results from evaluating the solar radiation model as well as the heating and cooling behavior of the room.

\subsection{Solar radiation model}

The case offers a frame for evaluating the calculation of the solar radiation on a tilted surface, by providing results for solar radiation profiles for clear and cloudy days, for the south and west facing walls.
Figure 4 presents the solar radiation on the south side on a cloudy day, while figure 5 presents the results for a clear day. The profiles are similar to the ones obtained with the other programs, never going outside the maximal and minimal specified ranges.

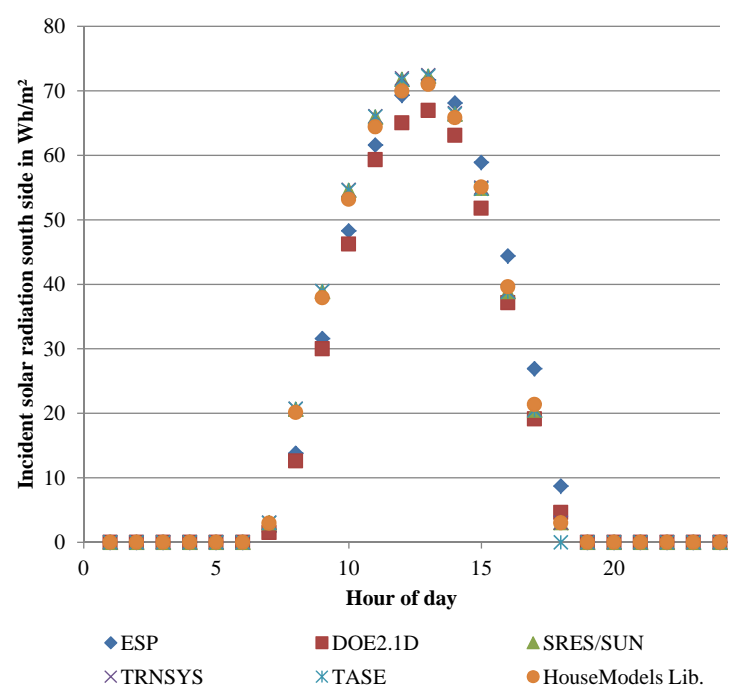

Figure 4: Comparison of hourly incident solar radiation for a cloudy day (March 5)

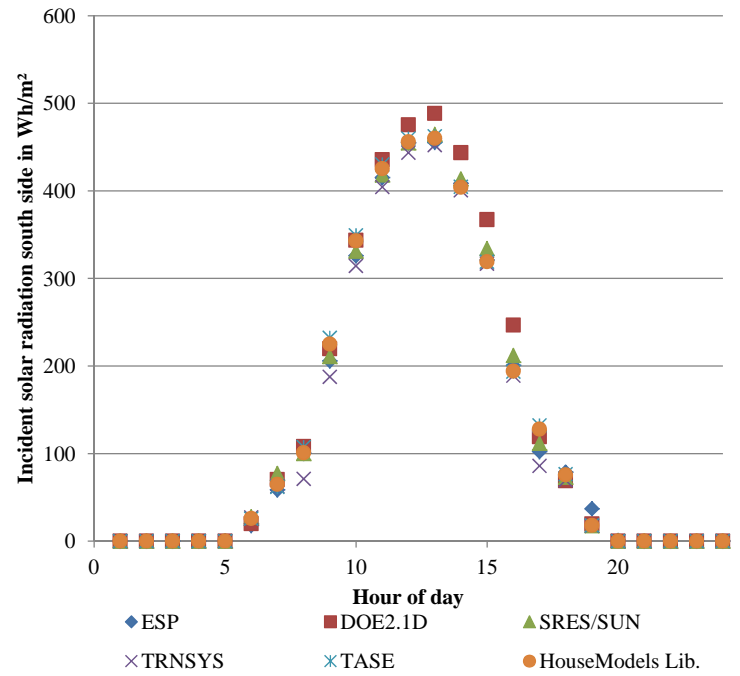

Figure 5: Comparison of hourly incident solar radiation for a cloudy day (July 27)

\subsection{Heating and Cooling behavior}

For evaluating the heating and the cooling behavior of the room, annual heating and cooling loads and annual hourly peaks as well as profiles for exemplary days are calculated. 
When considering the whole year, figure 6 presents a comparison of the heating and cooling loads for the whole year. The results for the annual heating load 5.081 MWh and cooling load 6.636 MWh are within the limits obtained with the other simulation tools.

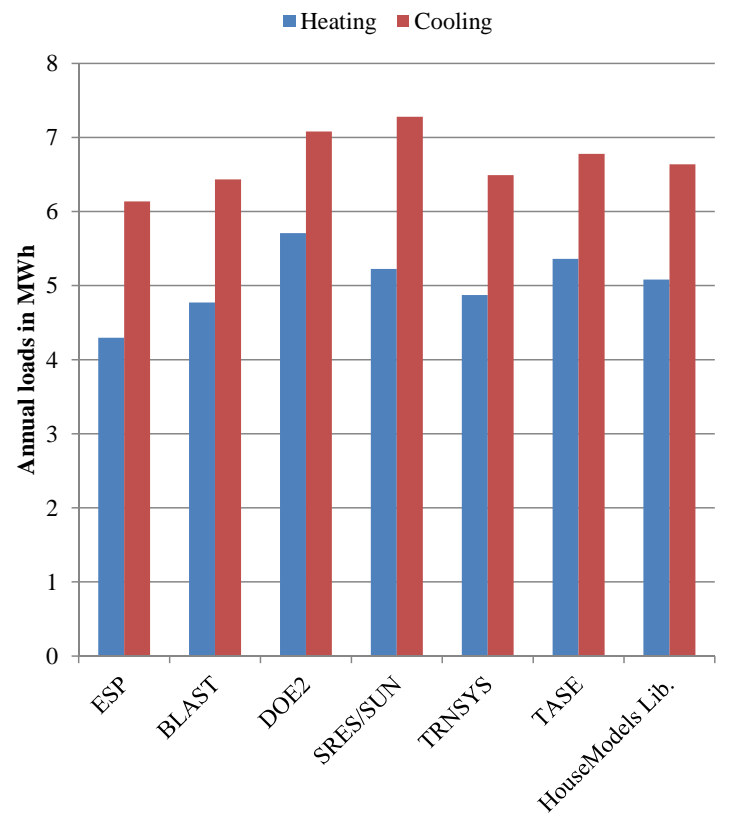

Figure 6: Comparison of annual heating and cooling loads

A more detailed look at the annual hourly heating and cooling peak loads (absolute value as well as day and hour when it occurs) is presented in tables 1 and 2 . The peak heating load of $4.132 \mathrm{~kW}$ occurring on the $4^{\text {th }}$ Jan and the peak cooling load of $6.2621 \mathrm{~kW}$ occurring on the $16^{\text {th }}$ Oct are similar to the results from the other programs: they are within the given limits and there is at least one software which gives the same time of occurence. The occurrence of the maximum cooling load on $16^{\text {th }}$ Oct is explained by the concurrence of a high ambient temperature of $22.2^{\circ} \mathrm{C}$ and a high incident solar radiation on the south side of $874 \frac{\mathrm{W}}{\mathrm{m}^{2}}$.

Table 1: Annual hourly integrated peak heating loads

\begin{tabular}{llll}
\hline Code Name & $\mathbf{k W}$ & Date & Hour \\
\hline ESP & 3.437 & 4 JAN & 5 \\
BLAST & 3.940 & 4 JAN & 5 \\
DOE2 & 4.045 & 4 JAN & 5 \\
SRES/SUN & 4.258 & 4 JAN & 2 \\
TRNSYS & 3.931 & 4 JAN & 6 \\
TASE & 4.354 & 4 JAN & 2 \\
HouseModels Lib. & 4.132 & 4 JAN & 2 \\
\hline
\end{tabular}

Table 2: Annual hourly integrated peak cooling loads

\begin{tabular}{llll}
\hline Code Name & kW & Date & Hour \\
\hline ESP & 6.149 & 17 OCT & 13 \\
BLAST & 5.965 & 16 OCT & 14 \\
DOE2 & 6.656 & 16 OCT & 13 \\
SRES/SUN & 6.827 & 16 OCT & 14 \\
TRNSYS & 6.486 & 16 OCT & 14 \\
TASE & 6.812 & 17 OCT & 13 \\
HouseModels Lib. & 6.261 & 16 OCT & 13 \\
\hline
\end{tabular}

Finally the test requires a comparison of the heating and cooling load profiles for the day with the highest heating load. Positive loads are heating loads, while negative loads are cooling loads. As shown in figure 7 the results obtained with our room model are similar to the ones from the other simulation tools.

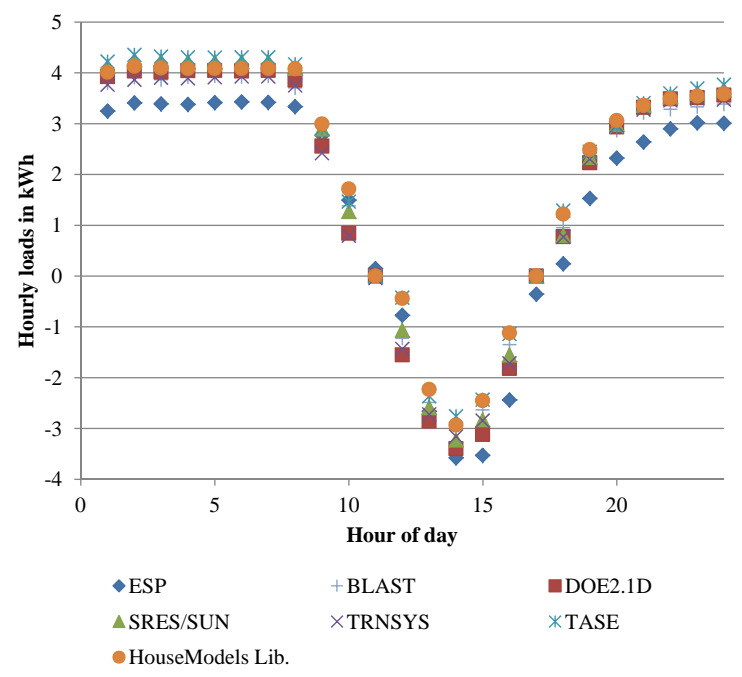

Figure 7: Comparison of hourly heating and cooling load profiles for Jan 4

\section{Conclusion}

In this paper we present our HouseModels library, which will be made available free of charge in summer 2014, as part of our contribution to the IEA Annex 60. The library contains complete standard house models for one and multi-family dwellings. The multi-family dwelling also contains a model for a single apartment. For each house type the models can be easily parameterized for different thermal masses and energy saving ordinances. A variation of the geometrical measure- 
ments is possible for the one family dwelling. This type of variations are useful when testing energy concepts and control strategies, as a robust system has to be able to adapt to different types of buildings.

Our motivation for creating this library is to bridge the gap between developers and users of Modelica for dynamic building system simulations. The models are easy to understand and use. Extra effort has been made to enrich the parameter window and to make the icons dynamic in regard to the chosen parameters. In order not to confuse beginners certain parameters have been set as protected and the parameterization of a room model can be done by specifying only a handful of parameters. The library is a useful tool for teaching, as students can learn to use Modelica with a focus on building systems while at the same time learning a few programming tricks.

The set of room types developed for the one family dwelling can, if necessary, be parameterized differently than the standard model or extended in order to build up specific house models.

As we try to keep the models as simple as possible and as detailed as needed in order to have good CPU-times for the simulations, a validation of the room models is currently on the way. In this paper we present first results obtained with case 600 of the ASHARE Standard 140. For all the required outputs our room model produced results within the minimum and maximum specified ranges. We plan on further evaluating the models with the whole suite of tests and improving the models if necessary.

\section{References}

[1] Müller, D., Hosseini Badakhshani, A. Gekoppelte Gebäude- und Anlagesimulation mit Modelica, BauSim, Vienna, 2010.

[2] IEA EBC Annex 60: New generation computational tools for building and community energy systems based on the Modelica and Functional Mockup Interface standards. Available: http://http://www.iea-annex60.org/.

[3] ANSI/ASHRAE Standard 140: Standard Method of Test for the Evaluation of Building Energy Analysis Computer Programs, American Society of Heating, Refrigerating and Air-Conditioning Engineers, Inc., Atlanta, 2007.

[4] Wetter, M. Zuo, W., Nouidui, T.S. Recent developments of the Modelica buildings library for building energy and control systems, in Proceesings of the 8th International Modelica Conference, Dresden, Germany, March 2011, 2011.

[5] Nytsch-Geusen, C., Huber, J., Ljubijankic, M. Modelica Building Systems - eine Modellbibliothek zur Simulation komplexer energietechnischer Gebaeudesysteme, BauSim, Berlin 2012.

[6] Nouidui, T.S., Phalak, K., Zuo, W., Wetter, M. Validation and application of the room model of the Modelica buildings library, in Proceesings of the 9th International Modelica Conference, Munich, Germany, September 20122012.

[7] Dymola 2014, Dassault Systems, 2014.

[8] Energieeinsparverordnung fuer Gebaeude (en: Energy Saving Ordinance), 2009.

[9] Cali, D., Streblow, R., Müller, D., Osterhage, T. Holistic Renovation and Monitoring of Residential Buildings in Proceedings of Rethink, renew, restart: ECEE 2013 summer study, 2013.

[10] Duffie, J., Beckmann, A. Solar engineering of thermal processes, Wiley, 2006. 